\title{
SEMI-PRECONTINUOUS FUNCTIONS AND PROPERTIES OF GENERALIZED SEMI-PRECLOSED SETS IN TOPOLOGICAL SPACES
}

\author{
G. B. NAVALAGI
}

Received 12 June 2000

\begin{abstract}
Andrijević (1986) introduced the class of semi-preopen sets in topological spaces. Since then many authors including Andrijevic have studied this class of sets by defining their neighborhoods, separation axioms and functions. The purpose of this paper is to provide the new characterizations of semi-preopen and semi-preclosed sets by defining the concepts of semi-precontinuous mappings, semi-preopen mappings, semi-preclosed mappings, semi-preirresolute mappings, pre-semipreopen mappings, and pre-semi-preclosed mappings and study their characterizations in topological spaces. Recently, Dontchev (1995) has defined the concepts of generalized semi-preclosed (gsp-closed) sets and generalized semi-preopen (gsp-open) sets in topology. More recently, Cueva (2000) has defined the concepts like approximately irresolute, approximately semi-closed, contra-irresolute, contra-semiclosed, and perfectly contra-irresolute mappings using semi-generalized closed (sg-closed) sets and semi-generalized open (sg-open) sets due to Bhattacharyya and Lahiri (1987) in topology. In this paper for gsp-closed (resp., gsp-open) sets, we also introduce and study the concepts of approximately semi-preirresolute (ap-sp-irresolute) mappings, approximately semi-preclosed (ap-semi-preclosed) mappings. Also, we introduce the notions like contra-semi-preirresolute, contra-semi-preclosed, and perfectly contra-semipreirresolute mappings to study the characterizations of semi-pre- $T_{1 / 2}$ spaces defined by Dontchev (1995).
\end{abstract}

2000 Mathematics Subject Classification: 54A05, 54C08, 54C10, 54D10, 54 G05.

1. Introduction. In the literature the notions of semi [14] (resp., pre [17], $\alpha$ [18])continuous mappings, semi [5] (resp., pre [17], $\alpha$ [18])-open mappings, semi [21] (resp., pre [12], $\alpha$ [18])-closed mappings, irresolute [10] (resp., preirresolute [23], $\alpha$-irresolute [16])-mappings were introduced and studied using semi [14] (resp., pre [17], $\alpha$ [20])open sets of and semi [6,9] (resp., pre [12], $\alpha$ [16, 18])-closed subsets of $X$. In this paper, we introduce the concepts of semi-precontinuous mappings, semi-preopen mappings, semi-preclosed mappings, semi-preirresolute mappings, pre-semipreopen mappings, and pre-semi-preclosed mappings and study their characterizations in topological spaces using the semi-preopen sets and semi-preclosed sets due to D. Andrijevic [3] (note that $\beta$-open sets in [1] are the same as the semi-preopen sets of D. Andrijevic [3]). Recently, in [11], Julian Dontchev has defined the concepts of generalized semi-preclosed (gsp-closed) sets and generalized semi-preopen (gsp-open) sets in topology. In this paper, using these sets, we also introduce and study the concepts of approximately semi-preirresolute mappings, and approximately semi-preclosed 
mappings. Also, we introduce the notions of contra-semi-preirresolute, contrasemi-preclosed, and perfectly contra-semi-preirresolute mappings to study the characterizations of semi-pre- $T_{1 / 2}$ spaces defined by Julian Dontchev in [11].

2. Preliminaries. Throughout the present paper, the sets $X, Y, Z$ always mean topological spaces and $f: X \rightarrow Y$ represents a single-valued function on which no separation axioms are assumed unless explicitly stated. Let $A$ be a subset of a space $X$. The closure of $A$ and the interior of $A$ are denoted by $\operatorname{cl} A$ and int $A$, respectively. The subset $A$ of $X$ is said to be (i) preopen [17] if $A \subset \operatorname{intcl} A$, (ii) semi-open [14] if $A \subset \operatorname{clint} A$, (iii) semi-preopen [3] if $A \subset \operatorname{clintcl} A$, and (iv) $\alpha$-open [20] set if $A \subset \operatorname{intclint} A$. The family of all preopen (resp., semi-open, semi-preopen, and $\alpha$-open) sets of $X$ is denoted by $\mathrm{PO}(X)$ (resp., $\mathrm{SO}(X), \mathrm{SPO}(X)$, and $\alpha(X)$ ). The family of all preopen (resp., semi-preopen) sets of $X$ containing a point $x$ is denoted by $\operatorname{PO}(x)$ (resp., $\operatorname{SPO}(x)$ ). The complement of a semi-preopen (resp., preopen, semi-open, and $\alpha$-open) set is called semi-preclosed (resp., preclosed, semi-closed, and $\alpha$-closed) set of $X$. The family of all semi-preclosed subsets of $X$ is denoted by $\operatorname{SPF}(X)$. A set $M_{x} \subset X$ is said to be semi-preneighborhood [19] of a point $x$ in $X$ if and only if there exists a semipreopen set $A$ containing $x$ such that $A \subset M_{x}$, the union of all semi-preopen sets that are contained in $A$ is called the semi-preinterior [3] of $A$ and is denoted by $\left(A_{S}\right)_{*}$ in [19] and the set $\cap\{F \subset X \mid A \subset F$ and $F$ is semi-preclosed set in $X\}$ is called the semipreclosure [3] of $A$ and is denoted by $(A s)^{*}$ in [19]. A mapping $f: X \rightarrow Y$ said to be (i) semi-continuous [14] if $f^{-1}(A) \in \mathrm{SO}(X)$ for every open set $A$ in $Y$, (ii) pre-continuous [17] if $f^{-1}(A) \in \mathrm{PO}(X)$ for every open set $A$ in $Y$, (iii) semi-open [5] if $f(A) \in \mathrm{SO}(Y)$ for every open set $A$ in $X$, (iv) preopen [17] if $f(A) \in \mathrm{PO}(Y)$ for every open set $A$ in $X$, (v) irresolute [10] if $f^{-1}(U) \in \mathrm{SO}(X)$ for every $U \in \mathrm{SO}(Y)$, (vi) preirresolute [23] if $f^{-1}(U) \in \mathrm{PO}(X)$ for every $U \in \mathrm{PO}(Y)$, (vii) pre-semiopen [10] if $f(A) \in \mathrm{SO}(Y)$ for every $A \in \mathrm{SO}(X)$, (viii) pre-semiclosed [22] if $f(A)$ is a semi-closed set in $Y$, for every semiclosed set $A$ of $X$, (ix) semi-closed [6] if $f(A)$ is semi-closed in $Y$ for each closed set $A$ in $X$ and $X$ preclosed [16] if $f(A)$ is preclosed in $Y$ for every closed set $A$ in $X$. More recently in [9], M. Caldas Cueva has defined the following notions: a map $f: X \rightarrow Y$ is called (i) approximately irresolute (written as ap-irresolute) if $s \operatorname{cl} F \subseteq f^{-1}(O)$, whenever $O$ is a semi-open subset of $Y, F$ is sg-closed subset of $X$, and $F \subseteq f^{-1}(O)$, (ii) approximately semi-closed (written as ap-semiclosed) map if $f(B) \subseteq s \operatorname{int} A$, whenever $A$ is a sg-open subset of $Y, B$ is a semi-closed subset of $X$, and $f(B) \subseteq A$, (iii) contrairresolute if $f^{-1}(O)$ is semi-closed in $X$ for each $O \in \mathrm{SO}(Y)$, (iv) contra-presemiclosed if $f(B) \in \mathrm{SO}(Y)$ for each semi-closed set $B$ of $X$, and (v) perfectly contra-irresolute if the inverse of every semi-open set in $Y$ is semi-clopen in $X$.

3. Semi-precontinuous functions. In this section, we introduce new weaker forms of continuity using semi-preopen sets and obtain their properties.

Definition 3.1. A function $f: X \rightarrow Y$ is called semi-precontinuous if the inverse image of each open set in $Y$ is a semi-preopen set in $X$.

Note 3.2. Every continuous function is a semi-precontinuous function but not the converse; which is verified by the following example. 
EXAMPLE 3.3. Let $X=\{a, b, c\}, \tau_{1}=\{\varnothing,\{a\},\{b\},\{a, b\}, X\}, Y=\{1,2,3,4\}, \tau_{2}=$ $\{\varnothing,\{1\},\{1,2\},\{1,2,3\}, Y\}$. A function $f: X \rightarrow Y$ is defined by $f(a)=1, f(b)=3, f(c)=2$. Here $\operatorname{SPO}\left(\tau_{1}\right)=\{\varnothing,\{a\},\{b\},\{a, b\},\{a, c\},\{b, c\}, X\}$. Then $f$ is semi-precontinuous. But $f$ is not continuous since $f^{-1}(\{1,2\})=\{a, c\}$, which is not a $\tau_{1}$-open set.

THEOREM 3.4. Let $f: X \rightarrow Y$ be a single-valued function, where $X$ and $Y$ are topological spaces. Then the following are equivalent:

(i) The function $f$ is semi-precontinuous.

(ii) For each point $p \in X$ and each open set $V$ in $Y$ with $f(p) \in V$, there is a SPO set $U$ in $X$ such that $p \in U, f(U) \subseteq V$.

(iii) The inverse of each closed set is semi-preclosed.

(iv) For each $x \in X$, the inverse of every neighborhood of $f(x)$ is a semipreneighborhood of $x$.

(v) For each $x \in X$ and each neighborhood $N_{x}$ of $f(x)$, there is a semipreneighborhood $V$ of $x$ such that $f(V) \subseteq N_{x}$.

(vi) For each subset $A$ of $X, f\left[\left(A_{s}\right)^{*}\right] \subseteq \operatorname{cl}[f(A)]$.

(vii) For each subset $B$ of $Y,\left(\left(f^{-1}(B)\right)_{s}\right)^{*} \subseteq f^{-1}(\operatorname{cl}(B))$.

Proof. (i) $\Leftrightarrow$ (ii). Let $f(p) \in V$ and $V \subset Y$ an open set, then $p \in f^{-1}(V) \in \operatorname{SPO}(\tau)$; since $f$ is semi-precontinuous. Let $U=f^{-1}(V)$, then $p \in U$ and $f(U) \subset V$.

Conversely, let $V$ be open in $Y$ and $p \in f^{-1}(V)$ then $f(p) \in V$, there exists a $U_{p} \in$ $\operatorname{SPO}(\tau)$ such that $p \in U_{p}$ and $f\left(U_{p}\right) \subset V$. Then $p \in U_{p} \subset f^{-1}(V)$ and $f^{-1}(V)=\cup U_{p}$, but by Note 3.2, $f^{-1}(V) \in \operatorname{SPO}(\tau)$, which implies that $f$ is semi-precontinuous.

(i) $\Leftrightarrow$ (iii). Assume $f$ is semi-precontinuous. Let $B$ be a closed subset of $Y$. Then $Y-B$ is open in $Y$ and $f^{-1}(Y-B)=X-f^{-1}(B) \in \operatorname{SPO}(\tau)$, which implies that $f^{-1}(B)$ is sp-closed.

Conversely, assume (iii). Let $G$ be an open set in $Y$ then $Y-G$ is a closed set in $Y$. Then $f^{-1}(Y-G)=X-f^{-1}(G)$. Hence $f^{-1}(G)$ is SPO in $X$ which implies that $f$ is semi-precontinuous.

(iii) $\Rightarrow$ (iv). Assume (iii) for $x \in X$, let $V$ be the neighborhood of $f(x)$ then $f(x) \in W \subset$ $V$, where $W=Y-F$ and $F$ is closed in $Y$. Consequently, $f^{-1}(W)$ is a semi-preopen set in $X$. Since $f^{-1}(W)=f^{-1}(Y-F)=X-f^{-1}(F), f^{-1}(F)$ is sp-closed by hypothesis, and $x \in f^{-1}(W) \subset f^{-1}(V)$. Then by definition, $f^{-1}(V)$ is a semi-preneighborhood of $X$.

(iv) $\Rightarrow(\mathrm{v})$. Let $x \in X$ and $N_{x}$ be a neighborhood of $f(x)$. Then $V=f^{-1}\left(N_{x}\right)$ is a sp-nhd of $x$ and $f(V)=f\left(f^{-1}\left(N_{x}\right)\right) \subset N_{x}$.

(v) $\Rightarrow$ (ii). For $x$ in $X$, let $W$ be an open set containing $f(x)$. Then $W$ is a neighborhood $V$ of $x$ such that $x \in V$ and $f(V) \subset W$. Hence there exists a semi-preopen set $A$ in $X$ such that $x \in A \subset V$, consequently, $f(A) \subset f(V) \subset W$.

(iii) $\Leftrightarrow$ (vi). Suppose that (iii) holds and let $A$ be a subset of $X$. Since $A \subset f^{-1}(f(A))$, we have $A \subset f^{-1}[\operatorname{cl}(f(A))]$. Now, $\operatorname{cl} f(A)$ is a closed set in $Y$ and hence $f^{-1}\{\operatorname{cl}(f(A))\}$ is a sp-closed set containing $A$, consequently, $\left(A_{s}\right)^{*} \subset f^{-1}\{\operatorname{cl}(f(A))\}$. Then, $f\left[\left(A_{s}\right)^{*}\right] \subset$ $f f^{-1}\{\operatorname{cl}(f(A))\} \subset \operatorname{cl}[f(A)]$. Conversely, suppose that (vi) holds for any subset $A$ of $X$. Let $F$ be a closed subset of $Y$. Then,

$$
f\left[\left(\left(f^{-1}(F)\right)_{s}\right)^{*}\right] \subset \operatorname{cl}\left(f\left(f^{-1}(F)\right)\right) \subset \operatorname{cl} F=F
$$

which implies that $\left(\left(f^{-1}(F)\right)_{s}\right)^{*} \subset f^{-1}(F)$. Consequently, the inverse of a closed set is semi-preclosed. 
(vi) $\Leftrightarrow$ (vii). Obvious.

Recall that a subset $A$ of $X$ is called regular open if $A=\operatorname{int} \operatorname{cl} A$, and that a mapping $f: X \rightarrow Y$ is called almost continuous [24] (written as a.c.S) if the inverse image of each regular open set is open.

LEMMA 3.5. If $f: X \rightarrow Y$ is an open, a.c.S, and preirresolute map then $f^{-1}(B) \in$ $\operatorname{SPO}(X)$ for each $B \in \mathrm{SPO}(Y)$.

Proof. Suppose $B$ is an arbitrary semi-preopen set in $Y$. Then there exists a preopen set $V$ in $Y$ such that $V \subset B \subset \operatorname{cl} V$. Now, by the a.c.Sness of $f$, we have $f^{-1}(V) \subset$ $f^{-1}(B) \subset f^{-1}(\operatorname{cl} V)=\operatorname{cl}\left(f^{-1}(V)\right)$ [2]. Since $f$ is preirresolute and $V$ is a preopen set in $Y, f^{-1}(V) \in \mathrm{PO}(X)$. Hence, $f^{-1}(V) \subset f^{-1}(B) \subset\left(\operatorname{cl} f^{-1}(V)\right)$ which implies that $f^{-1}(B) \in \operatorname{SPO}(X)$.

LEMMA 3.6. If $f: X \rightarrow Y$ is an open, a.c.S, and preirresolute mapping then the inverse image of each semi-preclosed set of $Y$ is a semi-preclosed set in $X$.

The proof is similar to Lemma 3.5.

THEOREM 3.7. Let $f: X \rightarrow Y$ be a mapping. Then the following are equivalent:

(i) The mapping $f$ is semi-precontinuous.

(ii) For each subset $G$ of $Y, f^{-1}(\operatorname{int} G) \subset\left(\left(f^{-1}(G)\right)_{s}\right)^{*}$.

Proof. (i) $\Rightarrow$ (ii). Let $G$ be any subset of $Y$. Then $\operatorname{int} G$ is an open set in $Y$ and $f^{-1}$ (int $G$ ) is a semi-preopen set in $X, f$ is semi-precontinuous. As $f^{-1}$ (int $\left.G\right) \subset f^{-1}(G)$, then $f^{-1}($ int $G) \subset\left(\left(f^{-1}(G)\right)_{s}\right)_{*}$.

The following lemma is proved in [3].

LEMMA 3.8. If $U$ is open and $A$ is semi-preopen then $U \cap A$ is a semi-preopen set.

LEMmA 3.9. Let $A$ be a semi-preopen set in a space $X$ and suppose $A \subset B \subset \operatorname{cl} A$, then $B$ is a semi-preopen set.

Proof. Since $A$ is a semi-preopen set in $X$, then there exists a preopen set $U$ in $X$ such that $U \subset A \subset \operatorname{cl} U$. As $A \subset B, U \subset A \subset B$ implies that $U \subset B$. Also, $\operatorname{cl} A \subset \operatorname{cl}(\operatorname{cl} U)=\operatorname{cl} U$, and thus, $B \subset \operatorname{cl} U$. Hence $U \subset B \subset \operatorname{cl} U$, which implies that $B$ is a semi-preopen set.

THEOREM 3.10. If $A \subset X_{0} \subset X$ and $X_{0} \in \operatorname{SPO}(X)$. Then $A \in \operatorname{SPO}(X)$ if and only if $A \in \operatorname{SPO}\left(X_{0}\right)$.

Proof. Necessity: since $A \in \operatorname{SPO}(X)$, then there exists a preopen set $U \subset X$ such that $U \subset A \subset \mathrm{cl} U$. Let $\mathrm{cl}_{X}$ and $\mathrm{cl}_{X_{0}}$ denote, respectively, the closure operator in $X$ and $X_{0}$. Now, $U \subset X_{0}$ as $X_{0} \subset X$. Then, $U=U \cap X_{0} \subset A \cap X_{0} \subset X_{0} \cap \mathrm{cl}_{X} U$, or $U \subset A \subset \mathrm{cl}_{X_{0}} U$, since $U=U \cap X_{0}$ and $U$ is preopen in $X_{0}$ by [18, Lemma 2.3], then it follows that $A \in \operatorname{SPO}\left(X_{0}\right)$.

The converse is easy and hence is omitted.

LEMMA 3.11 (see [19]). For every $A \in \operatorname{SPO}(X), \operatorname{cl} A=\operatorname{clint} c l A$.

LEMMA 3.12. $A$ is a semi-preopen set and $A \neq \varnothing$. Then, $\operatorname{int} \operatorname{cl} A \neq \varnothing$. 
Proof. Let $A$ be a semi-preopen set such that $A \neq \varnothing$. Then by Lemma 3.11, $\operatorname{cl} A=$ $\operatorname{clint} \operatorname{cl} A$. If $\operatorname{int} \operatorname{cl} A=\varnothing$ then $\operatorname{cl} A=\varnothing$ implies $A=\varnothing$, which is in contradiction to the hypothesis. Hence, int $\operatorname{cl} A \neq \varnothing$.

THEOREM 3.13. If $f: X \rightarrow Y$ is a semi-precontinuous map and $X_{0}$ is an open set in $X$, then the restriction $f / X_{0}: X_{0} \rightarrow Y$ is semi-precontinuous.

Proof. Since $f$ is semi-precontinuous, for any open set $V$ in $Y, f^{-1}(V)$ is a semipreopen set in $X$. Hence by Lemma 3.8, $f^{-1}(V) \cap X_{0}$ is a semi-preopen set in $X$ since $X_{0}$ is an open set. Therefore, by Theorem 3.10, $\left(f / X_{0}\right)^{-1}(V)=f^{-1}(V) \cap X_{0}$ is a semipreopen set in $X_{0}$, which implies that $f / X_{0}$ is semi-precontinuous.

We give the following definition.

Definition 3.14. A cover $u=\left\{U_{\alpha} \mid \alpha \in \Delta\right\}$ of subsets of $X$ is called a sp-cover if $U_{\alpha}$ is semi-preopen for each $\alpha \in \Delta$.

Now, we prove the following theorem.

THEOREM 3.15. Let $f: X \rightarrow Y$ be a map and $\left\{A_{\alpha} \mid \alpha \in \Delta\right\}$ a sp-cover of $X$. If the restriction, $f / A_{\alpha}: A_{\alpha} \rightarrow Y$ is semi-continuous for each $\alpha \in \Delta$, then $f$ is semiprecontinuous.

Proof. Suppose $V$ is an arbitrary open set in $Y$. Then for each $\alpha \in \Delta$, we have $\left(f / A_{\alpha}\right)^{-1}(V)=f^{-1}(V) \cap A_{\alpha} \in \operatorname{SPO}\left(A_{\alpha}\right)$ since $f / A_{\alpha}$ is semi-precontinuous. Hence by Theorem 3.10, $f^{-1}(V) \cap A_{\alpha} \in \operatorname{SPO}(X)$ for each $\alpha \in \Delta$. But, we know that arbitrary union of semi-preopen sets is a semi-preopen set, thus, we obtain that $\cup_{\alpha \in \Delta}\left[f^{-1}(V) \cap\right.$ $\left.A_{\alpha}\right]=f^{-1}(V) \in \operatorname{SPO}(X)$. This implies that $f$ is a semi-precontinuous map.

LEMMA 3.16 (see [13]). Let $\left\{X_{\alpha} \mid \alpha \in \Delta\right\}$ be a family of topological spaces and $\Pi A_{\alpha}$ a subset of $\Pi X \alpha$, where $\Pi X_{\alpha}$ denotes the product space. Then,

(i) int $\Pi A_{\alpha}=\Pi \operatorname{int} A_{\alpha}$ if $A_{\alpha}=X_{\alpha}$ except for a finite number of $\alpha \in \Delta$ and $\Pi A_{\alpha} \neq \varnothing$,

(ii) $\operatorname{cl} \Pi A_{\alpha}=\Pi \operatorname{cl} A_{\alpha}$.

Now, in view of Lemma 3.16, one can prove the following lemma.

LEMMA 3.17. Let $X_{1}$ and $X_{2}$ be topological spaces. If $A_{i}$ is a semi-preopen set in $X_{i}$ for each $i=1,2$, then $A_{1} \times A_{2}$ is a semi-preopen set in the product space $X_{1} \times X_{2}$.

The following theorem proved in [22] is the generalization of Lemma 3.17.

THEOREM 3.18 (see [22]). Let $\left\{X_{\alpha} \mid \alpha \in \Delta\right\}$ be a family of topological spaces, $X=$ $\Pi X_{\alpha}$, the product space, and $A=\prod_{j=1}^{n} A_{\alpha_{j}} \times \prod_{\alpha \neq \alpha_{j}} X_{\alpha}$ a nonempty subset of $X$, where $n$ is a positive integer. Then, $A_{\alpha_{j}} \in \operatorname{SPO}\left(X_{\alpha_{j}}\right)$ for each $j(1 \leq j \leq n)$ if and only if $A \in \operatorname{SPO}(X)$.

Next, we prove the following theorem.

THEOREM 3.19. Let $X_{i}$ and $Y_{i}$ be topological spaces and $f_{i}: X_{i} \rightarrow Y_{i}$ be a semiprecontinuous mapping for $i=1,2$. Then a mapping $f: X_{1} \times X_{2} \rightarrow Y_{1} \times Y_{2}$ defined by putting $f\left(\left(x_{1}\right),\left(x_{2}\right)\right)$ is semi-precontinuous. 
Proof. Let $O_{1} \times O_{2} \subset Y_{1} \times Y_{2}$, where $O_{i}$ is open in $Y_{i}$ for $i=1$, 2 . Then, $f^{-1}\left(O_{1} \times O_{2}\right)$ $=f^{-1}\left(O_{1}\right) \times f^{-1}\left(O_{2}\right)$. But $f^{-1}\left(O_{1}\right), f^{-1}\left(O_{2}\right)$ are semi-preopen sets in $X_{1}$ and $X_{2}$, respectively, then $f^{-1}\left(O_{1}\right) \times f^{-1}\left(O_{2}\right)$ is semi-preopen in $X_{1} \times X_{2}$ by Lemma 3.17. Now, if $O$ is any open set in $Y_{1} \times Y_{2}$, then $f^{-1}(O)=f^{-1}\left(\cup O_{\alpha}\right)$, where $O_{\alpha}$ is of the form $O_{\alpha_{1}} \times O_{\alpha_{2}}$. Then $f^{-1}(O)=\cup f^{-1}\left(O_{\alpha}\right)$, which is a semi-preopen set since arbitrary union of semi-preopen sets is a semi-preopen set. Hence by arguing as above, $f^{-1}\left(O_{1} \times O_{2}\right)$ is a semi-preopen set in $X_{1} \times X_{2}$. Hence $f$ is semi-precontinuous.

The following theorem is the generalization of Theorem 3.19, which can be proved in view of Theorems 3.18 and 3.19 .

THEOREM 3.20. Let $\left\{X_{\alpha} \mid \alpha \in \Delta\right\}$ and $\left\{Y_{\alpha} \mid \alpha \in \Delta\right\}$ be any two families of topological spaces with the same index set $\Delta$. For each $\alpha \in \Delta$, let $f_{\alpha}: X_{\alpha} \rightarrow Y_{\alpha}$ be a mapping, then a mapping $f: \Pi X_{\alpha} \rightarrow \Pi Y_{\alpha}$ defined by $f\left(\left(x_{\alpha}\right)\right)=\left(f_{\alpha}\left(x_{\alpha}\right)\right)$ is semi-precontinuous if and only if $f_{\alpha}$ is semi-precontinuous for each $\alpha \in \Delta$.

4. Semi-preopen functions. We give the following definition.

Definition 4.1. A function $f:(X, \tau) \rightarrow(Y, \sigma)$ is called semi-preopen if the image of each open set in $X$ is a semi-preopen set in $Y$.

Note that every open map is semi-preopen but not the converse, which is shown by the following example.

EXAMPLE 4.2. Let $X=\{p, q, r\}, \tau=\{\varnothing,\{p\},\{p, q\}, X\}, Y=\{a, b, c\}, \sigma=\{\varnothing,\{a\}$, $\{b\}\{a, b\}, Y\}$. Then it is clear that $\tau$ and $\sigma$ are topologies on $X$ and $Y$, respectively. If $f:(X, \tau) \rightarrow(Y, \sigma)$ is a map defined by $f(p)=a, f(q)=c$, and $f(r)=b$. It is clear that $f$ is a semi-preopen mapping but it is not open mapping since $f(\{p, q\})=\{a, c\} \notin \sigma$. Also, note that every preopen (resp., semi-open) map is a semi-preopen map.

We recall that a mapping $f: X \rightarrow Y$ is called semi-preopen (in the sense of Cammaroto and Noiri [8]) if $f(U) \in \mathrm{SPO}(Y)$ for each $U \in \mathrm{SO}(X)$. Clearly, every semipreopen map (in the sense of Cammaroto and Noiri [8]) is a semi-preopen map as given by Definition 4.1 .

We recall the following lemma.

LEMMA 4.3 (see [8]). The following are equivalent for a subset $A$ of a space $X$ :

(i) $A \in \operatorname{SPO}(X)$.

(ii) $A \subset \operatorname{cl}(\operatorname{int}(\operatorname{cl}(A)))$.

(iii) $A \subset \operatorname{sint}(s \operatorname{cl}(A))$.

THEOREM 4.4. A mapping $f: X \rightarrow Y$ is semi-preopen if and only if for every subset $A \subset X, f(\operatorname{int} A) \subset \operatorname{sint}(s \operatorname{cl}(A))$.

Proof. Let $f$ be a semi-preopen map. We have $f(\operatorname{int} A) \subset f(A)$ for each $A \subset X$ and by hypothesis $f(\operatorname{int} A$ ) is a semi-preopen set in $Y$ and by Lemma 4.3, $f(\operatorname{int} A) \subset$ $s \operatorname{int} s \mathrm{cl}(f(A))$. Conversely, let the given condition holds true and $G$ any open set in $X$. Then $f(G)=f(\operatorname{int} G) \subset \operatorname{sint} s \operatorname{cl}(f(G))$ which implies that $f(G) \subset \operatorname{sint} s \operatorname{cl}(f(G))$. Thus, $f(G)$ is a semi-preopen set in $Y$ by Lemma 4.3 and hence $f$ is a semi-preopen map. 
THEOREM 4.5. Let $X, Y$, and $Z$ be three topological spaces and let $f: X \rightarrow Y$ and $g: Y \rightarrow Z$ be two mappings with $g \circ f: X \rightarrow Z$ is a semi-preopen mapping. Then,

(i) If $f$ is continuous and surjective, then $g$ is semi-preopen.

(ii) If $g$ is preopen, preirresolute, and injective, then $f$ is semi-preopen.

Proof. (i) Let $V$ be an arbitrary open set in $Y$. Since $g \circ f$ is semi-preopen and $f$ is surjective then $g(V)=g \circ f\left\{f^{-1}(V)\right\}$ is a semi-preopen set in $Z$. This shows that $g$ is a semi-preopen map.

(ii) Since $g$ is injective, we remark that $f(A)=g^{-1}[g(f(A))]$ for every subset $A$ of $X$. Let $U$ be an arbitrary open set in $X$, then by hypothesis, $g \circ f(U)$ is a semi-preopen set in $Z$. Then by Note 3.2, we have $f(U)=g^{-1}(g \circ f(U)) \in \operatorname{SPO}(Y)$ which implies that $f(U)$ is a semi-preopen set in $Y$. Hence, $f$ is a semi-preopen map.

5. Semi-preclosed functions. We recall the following definition.

DEFINITION 5.1. A function $f: X \rightarrow Y$ is called semi-preclosed if the image of each closed set in $X$ is a semi-preclosed set in $Y$ [22].

Note that every closed map is semi-preclosed but not the converse, which is shown by the following example.

EXAMPLE 5.2. Let $X=\{a, b, c\}, \tau=\{\varnothing,\{a\},\{a, b\},\{a, c\}, X\}$, and $\sigma=\{\varnothing,\{a\}$, $\{a, b\}, X\}$. Then $f:(X, \tau) \rightarrow(X, \sigma)$ is the identity mapping. Here, clearly $\tau$ and $\sigma$ are topologies on $X$. And, the $\tau$-closed sets are $\{\varnothing,\{b\},\{c\},\{b, c\}, X\}$, the $\sigma$-closed sets are $\{\varnothing,\{c\},\{b, c\}, X\}$ and $\operatorname{SPF}(\sigma)=\{\varnothing,\{b\},\{c\},\{b, c\}, X\}$.

Clearly, $f$ is a semi-preclosed map but it is not closed since $f(\{b\})=\{b\}$ which is not a $\sigma$-closed set. Hence the example.

Note that every preclosed map (resp., semi-closed map) is a semi-preclosed map. Thus, we state the following theorem.

THEOREM 5.3. A map $f: X \rightarrow Y$ is semi-preclosed if $f$ is both a semi-closed and a preclosed map.

THEOREM 5.4. Let $f:(X, \tau) \rightarrow(Y, \sigma)$ be a mapping from a space $(X, \tau)$ into a space $(Y, \sigma)$. Then $f$ is semi-preclosed if and only if $\left((f(A))_{s}\right)^{*} \subset f\left(\left(A_{S}\right)^{*}\right)$, for each set $A$ of $(X, \tau)$.

Proof. Let $f$ be a semi-preclosed map and $A$ any subset of $X$. Then $f\left(\left(A_{s}\right)^{*}\right) \in$ $\operatorname{SPF}(Y)$. As $f(A) \subset f\left(\left(A_{s}\right)^{*}\right)$, it follows that $(f(A))_{s}^{*} \subset f\left(\left(A_{s}\right)^{*}\right)$.

Conversely, assume that $F \in \operatorname{SPF}(X)$. Then $f(F)=f\left(\left(F_{S}\right)^{*}\right) \supset\left((f(F))_{s}\right)^{*}$, thus we obtain that $(f(F))_{s}^{*}=f(F)$. Hence, $f$ is a semi-preclosed map.

Next, we give the following definition.

DefinITION 5.5. A mapping $f: X \rightarrow Y$ is said to be $s$-preclosed if $f(F) \in \operatorname{SPF}(Y)$ for each semi-closed set $F$ of $X$.

Clearly, every s-preclosed map is semi-preclosed. But semi-closed, preclosed, and $s$-preclosed maps are, respectively, independent of each other. 
EXAMPLE 5.6. Let $X=\{p, q, r\}, \tau=\{\varnothing,\{r\},\{q, r\}, X\}$, and $\sigma=\{\varnothing,\{r\},\{p, r\}$, $\{q, r\}, X\}$. Let $f:(x, \tau) \rightarrow(X, \sigma)$ be a mapping defined by $f(p)=q, f(q)=r$, and $f(r)=r$. Then clearly, $f$ is $s$-preclosed but $f$ is not a preclosed map since $f(\{p, q\})=$ $\{q, r\}$ which not preclosed in $(X, \sigma)$. This shows that $s$-preclosed and preclosed maps are independent of each other.

Recall the following lemma.

LEMмA 5.7 (see [22]). If $f: X \rightarrow Y$ is semi-preclosed, then for each subset $V$ of $Y$ and each open set $U$ of $X$ containing $f^{-1}(V)$, there exists $W \in \operatorname{SPO}(Y)$ such that $V \subset W$ and $f^{-1}(W) \subset U$.

Now, we can prove it for $s$-preclosed maps in the following theorem.

THEOREM 5.8. If $f: X \rightarrow Y$ is s-preclosed, then for each subset $V$ of $Y$ and each semi-open set $U$ of $X$ containing $f^{-1}(V)$, there exists $W \in \operatorname{SPO}(Y)$ such that $V \subset W$ and $f^{-1}(W) \subset U$.

\section{Semi-preirresolute functions}

Definition 6.1. A function $f: X \rightarrow Y$ is called semi-preirresolute if the inverse image of each semi-preopen set in $Y$ is a semi-preopen set in $X$.

Note that every semi-preirresolute map is semi-precontinuous but not the converse, which is shown by the following example.

EXAMPLE 6.2. Let $X=\{a, b, c, d\}$ and $\tau=\{\varnothing,\{a\},\{b\},\{c\},\{a, b\},\{a, c\},\{b, c\}$, $\{b, d\},\{a, b, c\},\{a, b, d\},\{b, c, d\}, X\} ; Y=\{m, n, l\}$, and $\sigma=\{\varnothing,\{m\}, Y\}$. Let $f: X \rightarrow Y$ be a mapping defined by $f(a)=m, f(b)=f(c)=l$, and $f(d)=n$.

Then, clearly $f$ is semi-precontinuous but it is not a semi-preirresolute map since $f^{-1}(\{m, n\})=\{a, d\}$ which is not a semi-preopen set in $(Y, \sigma)$.

Next, we characterize the semi-preirresolute mappings in the following theorem.

THEOREM 6.3. The following statements are equivalent for a function $f: X \rightarrow Y$ :

(i) $f$ is semi-preirresolute.

(ii) For each point $x$ of $X$ and each semi-preneighborhood $V$ of $f(x)$, there exists a semi-preneighborhood $U$ of $x$ such that $f(U) \subseteq V$.

(iii) For each $x \in X$ and each $V \in \operatorname{SPO}(f(x))$, there exists $U \in \operatorname{SPO}(x)$ such that $f(U) \subseteq V$.

Proof. (i) $\Rightarrow$ (ii). Assume $x \in X$ and $V$ is a semi-preopen set in $Y$ containing $f(x)$. Since $f$ is a semi-preirresolute and let $W=f^{-1}(V)$ be a semi-preopen set in $X$ containing $x$ and hence $f(W) \subset f\left(f^{-1}(V)\right) \subset V$.

(ii) $\Rightarrow$ (iii). Assume that $V \subset Y$ is a semi-preopen set containing $f(x)$. Then by (ii), there exists a semi-preopen set $G$ such that $x \in G \subset f^{-1}(V)$. Therefore, $x \in f^{-1}(V) \subset$ $\operatorname{cl}\left(f^{-1}(V)\right)$. This shows that $\mathrm{cl}\left(f^{-1}(V)\right)$ is a semi-preneighborhood of $x$.

(iii) $\Rightarrow$ (i). Let $V$ be a semi-preopen set in $Y$, then $\mathrm{cl}\left(f^{-1}(V)\right)$ is semi-preneighborhood of each $x \in f^{-1}(V)$. Thus, for each $x$ is a semi-preinterior point of $\operatorname{cl}\left(f^{-1}(V)\right)$ which implies that $f^{-1}(V) \subset \operatorname{int} \operatorname{cl}\left(f^{-1}(V)\right) \subset \operatorname{clintcl}\left(f^{-1}(V)\right)$. Therefore, $f^{-1}(V)$ is a semipreopen set in $X$ and hence $f$ is a semi-preirresolute map. 
We state the following theorems.

THEOREM 6.4. If $f: X \rightarrow Y$ is a preopen and preirresolute mapping, then $f$ is a semi-preirresolute.

THEOREM 6.5. If $f: X \rightarrow Y$ is semi-preirresolute and $g: Y \rightarrow Z$ is semi-precontinuous, then $g \circ f$ is a semi-precontinuous map.

Recall the following theorem.

Theorem 6.6 (see [10, Theorem 1.1]). If $f: X \rightarrow Y$ is continuous and open, then $f^{-1}(\operatorname{cl} A)=\operatorname{cl}\left(f^{-1}(A)\right)$ for every subset $A$ of $Y$.

Now, we prove the following theorem.

THEOREM 6.7. Let $f: X \rightarrow Y$ be a continuous open and preirresolute mapping, then $f$ is a semi-preirresolute mapping.

Proof. Let $A \in \operatorname{SPO}(Y)$, then there exists a preopen set $U \subset Y$ such that $U \subset A \subset \operatorname{cl} U$. Then by Theorem 6.6, $f^{-1}(\operatorname{cl} U)=\operatorname{cl}\left(f^{-1}(U)\right)$. Also, we have

$$
f^{-1}(U) \subset f^{-11}(A) \subset f^{-1}(\operatorname{cl} U)=\operatorname{cl}\left(f^{-1}(U)\right) .
$$

Since $f$ is a preirresolute map, then $f^{-1}(U)$ is a preopen set in $X$, and hence $f^{-1}(A)$ is a semi-preopen set in $X$. Thus, $f$ is a semi-preirresolute map.

One can easily prove the following theorem.

THEOREM 6.8. A mapping $f: X \rightarrow Y$ is semi-preirresolute if and only if for every semi-preclosed set $F$ of $Y, f^{-1}(F)$ is a semi-preclosed set in $X$.

\section{Semi-prehomeomorphisms}

DeFinITION 7.1. A bijective mapping $f:(X, \tau) \rightarrow(Y, \sigma)$ from a space $X$ into a space $Y$ is called a semi-prehomeomorphism if both $f$ and $f^{-1}$ are semi-preirresolute mappings.

Now, we characterize the semi-prehomeomorphism in the following theorem.

THEOREM 7.2. Let $f:(X, \tau) \rightarrow(Y, \sigma)$ be a bijective mapping from a space $X$ into a space $Y$. Then the following are equivalent:

(i) $f$ is a semi-prehomeomorphism.

(ii) $f^{-1}$ is a semi-prehomeomorphism.

Proof. (i) $\Leftrightarrow$ (ii). Since $f$ is a bijective map, both $f$ and $f^{-1}$ are semi-preirresolute functions.

DEFINITION 7.3. A property which is preserved under semi-prehomeomorphism is said to be a semi-pretopological property.

Clearly every homeomorphism is semi-prehomeomorphism.

8. Pre-semipreopen functions. In this section, we introduce the notion of presemipreopen mappings analogous to pre-semiopen mappings [10]. 
DEFINITION 8.1. A function $f: X \rightarrow Y$ is called pre-semipreopen if the image of each semi-preopen set in $X$ is a semi-preopen set in $Y$.

Note that every pre-semipreopen map is semi-preopen but not the converse, which is shown by the following example.

EXAMPLE 8.2. Let $X=\{a, b, c\}, \tau=\{\varnothing,\{a\},\{a, b\}, X\}, Y=\{p, q, r\}$, and $\sigma=$ $\{\varnothing,\{p\},\{q\},\{p, q\},\{q, r\}, Y\}$. Assume, a function $f: X \rightarrow Y$ is defined by $f(a)=\{p\}$, $f(b)=\{q\}$, and $f(c)=\{r\}$. Then, clearly $f$ is a semi-preopen map but it is not presemipreopen since $f(\{a, c\})=\{p, r\} \notin \operatorname{SPO}(Y, \sigma)$.

REMARK 8.3. (i) A pre-semipreopen map need not be open.

(ii) An open map need not be pre-semipreopen.

THEOREM 8.4. If a mapping $f: X \rightarrow Y$ is pre-semipreopen then $\left((f(A))_{S}\right)_{*} \subset f\left(\left(A_{s}\right)_{*}\right)$ for every subset $A$ of $X$.

Proof. Suppose $f$ is a pre-semipreopen map and $A$ any arbitrary subset of $X$. Since $\left(A_{S}\right)_{*}$ is a semi-preopen set, $f\left(\left(A_{S}\right)_{*}\right)$ is a semi-preopen set in $Y$ as $f$ is a presemipreopen map. Hence, we obtain that $\left((f(A))_{s}\right)_{*} \subset f\left(\left(A_{s}\right)_{*}\right)$.

THEOREM 8.5. Let $f: X \rightarrow Y$ and $g: Y \rightarrow Z$ be two maps such that $g \circ f$ is a presemipreopen map. Then,

(i) If $f$ is a semi-preirresolute surjection, then $g$ is a pre-semipreopen map.

(ii) If $g$ is a semi-preirresolute injection, then $f$ is a pre-semipreopen map.

Proof. (i) Let $A$ be any semi-preopen set in $Y$. Since $f$ is a semi-preirresolute map, $f^{-1}(A)$ is a semi-preopen set in $X$. As $g \circ f$ is a pre-semipreopen map and $f$ is surjective, $g \circ f\left(f^{-1}(A)\right)=g(A)$, which is a semi-preopen set in $Z$. This implies that $g$ is a pre-semipreopen map.

(ii) As we claimed in (i), we can prove the second part easily.

\section{Pre-semi-preclosed functions}

DefinITION 9.1. A function $f: X \rightarrow Y$ is called pre-semi-preclosed if the image of each semi-preclosed set in $X$ is a semi-preclosed set in $Y$.

Note that every pre-semi-preclosed map is semi-preclosed but not the converse, which is shown by the following example.

EXAMPLE 9.2. Consider $(X, \tau),(Y, \sigma)$, and $f$ be as defined in Example 8.2. By taking complements, one can show that $f$ is a semi-preclosed map but not a presemi-preclosed map since $f(\{b\})=\{q\} \notin \operatorname{SPF}(Y, \sigma)$.

REMARK 9.3. Both closed and pre-semi-preclosed maps are independent of each other.

One can prove the following theorem similar to Lemma 5.7.

THEOREM 9.4. If a mapping $f: X \rightarrow Y$ is pre-semi-preclosed, then for each subset $B$ of $Y$ and each semi-preopen set $V$ in $X$ containing $f^{-1}(B)$, there exists a semi-preopen set $U$ in $Y$ containing $B$ such that $f^{-1}(U) \subset V$. 
REMARK 9.5. In Theorem 9.4, if $f$ is also a surjective map then, the only if part holds true.

We prove the following theorem.

THEOREM 9.6. Let $f: X \rightarrow Y$ and $g: Y \rightarrow Z$ be two maps such that $g \circ f$ is a presemi-preclosed map. Then,

(i) If $f$ is a semi-preirresolute surjection, then $g$ is a pre-semi-preclosed map.

(ii) If $g$ is a semi-preirresolute injection, then $f$ is a pre-semi-preclosed map.

Proof. We prove (ii) only. Suppose $A$ is an arbitrary semi-preclosed set in $X$. Since $g \circ f$ is a pre-semi-preclosed map, then $g \circ f(A)$ is a semi-preclosed set in Z . Since $g$ is a semi-preirresolute injective map, we have $g^{-1}(g \circ f(A))=f(A)$, which is a semi-preclosed set in $Y$. This shows that $f$ is pre-semi-preclosed.

Recall that a map $f: X \rightarrow Y$ is called $M$-preclosed [23] if the image of each preclosed set is a preclosed set.

Finally, we prove the following theorem.

THEOREM 9.7. If $f: X \rightarrow Y$ is a continuous $M$-preclosed injective map then $f$ is a pre-semi-preclosed map.

Proof. Let $f$ be a continuous $M$-preclosed injective map and $A$ a semi-preclosed set in $X$. Then, there exists a preclosed set $F$ in $X$ such that $\operatorname{int} F \subset A \subset F$ and so $f(\operatorname{int} F) \subset f(A) \subset f(F)$. Since $f$ is a continuous injective map, $\operatorname{int}(f(F)) \subset f(\operatorname{int} F)$ and also $f$ is a $M$-preclosed map, $f(F)$ is a preclosed set in $Y$. Then, we obtain that $\operatorname{int}(f(F)) \subset f(A) \subset f(F)$ which implies that $f(A)$ is a semi-preclosed set in $Y$. Thus, $f$ is a pre-semi-preclosed map.

10. Generalized semi-preclosed sets and their mappings. We recall the following definition.

Definition 10.1 (see [15]). A subset $A$ of a space $X$ is called a generalized closed set (written as $g$-closed) set if $\operatorname{cl} A \subseteq U$ whenever $A \subseteq U$ and $U$ is open.

Clearly, every closed set is a $g$-closed set. The complement of a $g$-closed set in $X$ is called generalized open, that is, $g$-open [15] set. So, every open set is a $g$-open set.

Definition 10.2 (see [4]). A subset $A$ of a space $X$ is called a semi-generalized closed set (written as sg-closed set) if $s \operatorname{cl} A \subseteq U$ whenever $A \subseteq U$ and $U$ is semi-open.

Clearly every semi-closed set is a sg-closed set. The complement of a sg-closed set is called a semi-generalized open set, that is, a sg-open set [4]. Every semi-open set is a sg-open set.

Definition 10.3 (see [11]). A subset $A$ of a space $X$ is called a generalized semipreclosed (written as gsp-closed) set if $\left(A_{s}\right)^{*} \subseteq U$ whenever $A \subseteq U$ and $U$ is open.

Clearly, every semi-preclosed set is a gsp-closed set. The complement of a gspclosed set is called generalized semi-preopen [11] (written as gsp-open). Every semipreopen set is a gsp-open set. The family of all gsp-closed (resp., gsp-open) sets of $X$ is denoted by $\operatorname{GSPF}(X)$ (resp., $\operatorname{GSPO}(X)$ ). 
Next, we define the following mappings.

DeFinITION 10.4. A function $f:(X, \tau) \rightarrow(Y, \sigma)$ is called approximately semipreirresolute (written as ap-sp-irresolute) if $\left(F_{S}\right)^{*} \subseteq f^{-1}(U)$, whenever $U$ is a semipreopen subset of $(Y, \sigma), F$ is a gsp-closed subset of $(X, \tau)$, and $F \subseteq f^{-1}(U)$.

Note that every semi-preirresolute (i.e., sp-irresolute) map is ap-sp-irresolute but not the converse, which is shown by the following theorem.

THEOREM 10.5. A function $f:(X, \tau) \rightarrow(Y, \sigma)$ is ap-sp-irresolute if $f^{-1}(U)$ is semipreclosed in $(X, \tau)$ for every $U \in \mathrm{SO}(Y, \sigma)$.

Proof. Let $F \subseteq f^{-1}(U)$, where $U \in \operatorname{SPO}(Y, \sigma)$ and $F$ is a gsp-closed subset of $(X, \tau)$. Therefore, we have $\left(F_{S}\right)^{*} \subseteq\left(\left(f^{-1}(U)\right)_{S}\right)^{*}=f^{-1}(U)$. This implies that $f$ is an ap-spirresolute.

THEOREM 10.6. Let $f:(X, \tau) \rightarrow(Y, \sigma)$ be a map from a topological space $(X, \tau)$ into a topological space $(Y, \sigma)$. If the semi-preopen and semi-preclosed subsets of $(X, \tau)$ coincide, then $f$ is ap-sp-irresolute if and only if $f^{-1}(U)$ is semi-preclosed in $(X, \tau)$ for every $U \in \operatorname{SPO}(Y, \sigma)$.

Proof. Suppose that $f$ is an ap-sp-irresolute. Let $A$ be an arbitrary subset of $X$ such that $A \subseteq U$ where $U \in \operatorname{SPO}(X)$. Then by hypothesis $\left(A_{s}\right)^{*} \subseteq\left(U_{s}\right)^{*}=U$. Thus, all subsets of $X$ are gsp-closed and hence all are gsp-open sets. Therefore, for any $O$ in $\mathrm{SPO}(Y), f^{-1}(O)$ is gsp-closed in $X$. Since $f$ is ap-sp-irresolute, $\left(\left(f^{-1}(O)\right)_{s}\right)^{*} \subseteq f^{-1}(O)$. This shows that $\left(\left(f^{-1}(O)\right)_{s}\right)^{*}=f^{-1}(O), f^{-1}(O)$ is a semi-preclosed set in $X$.

Converse follows from Theorem 10.5.

DeFinITION 10.7. A function $f:(X, \tau) \rightarrow(Y, \sigma)$ is called approximately semipreclosed (written as ap-sp-closed) if $f(B) \subseteq\left(A_{S}\right)^{*}$ whenever $A$ is a gsp-open subset of $(Y, \sigma), B$ is a semi-preclosed subset of $(X, \tau)$, and $f(B) \subseteq A$.

Note that every pre-semi-preclosed map is an ap-sp-closed map but not the converse, which is shown by the following theorem.

THEOREM 10.8. A function $f:(X, \tau) \rightarrow(Y, \sigma)$ is ap-semi-preclosed if $f(B) \in \operatorname{SPO}(Y, \sigma)$ for every semi-preclosed subset $B$ of $(X, \tau)$.

Proof. Let $f(B) \subseteq A$, where $B$ is a semi-preclosed subset of $(X, \tau)$ and $A$ is gspopen subset of $(Y, \sigma)$. Therefore, we have $\left((f(B))_{s}\right)^{*} \subseteq\left(A_{S}\right)^{*}$. Then $f(B) \subseteq\left(A_{S}\right)^{*}$ which implies that $f$ is an ap-semi-preclosed map.

The easy proof of the following theorem is omitted.

THEOREM 10.9. Let $f:(X, \tau) \rightarrow(Y, \sigma)$ be a map from a topological space $(X, \tau)$ into a topological space $(Y, \sigma)$. If the semi-preopen and semi-preclosed subsets of $(Y, \sigma)$ coincide, then $f$ is ap-semi-preclosed if and only if $f(B) \in \operatorname{SPO}(Y, \sigma)$, for every semipreclosed subset $B$ of $(X, \tau)$.

Also, we give the following definition.

DEFINITION 10.10. A function $f:(X, \tau) \rightarrow(Y, \sigma)$ is called contra-semi-preirresolute (written as contra-sp-irresolute) if $f^{-1}(O)$ is semi-preclosed in $(X, \tau)$ for each $O \in$ $\operatorname{SPO}(Y, \sigma)$. 
Note that every semi-preirresolute (i.e., sp-irresolute) map is ap-sp-irresolute but not the converse, which is shown by the following definition.

DEFINITION 10.11. A function $f:(X, \tau) \rightarrow(Y, \sigma)$ is called contra-presemipreclosed (written as contra-presp-closed) if $f(B) \in \operatorname{SPO}(Y, \sigma)$, for each semi-preclosed set $B$ of $(X, \tau)$.

Note that every pre-semi-preclosed map is an ap-sp-closed map but not the converse, which is shown by the following theorem.

THEOREM 10.12. Let $f:(X, \tau) \rightarrow(Y, \sigma)$ be a map. Then the following are equivalent:

(i) $f$ is contra-sp-irresolute.

(ii) The inverse image of each semi-preclosed set in $Y$ is a semi-preopen set in $X$.

Next, we give the following definition.

Definition 10.13. A subset of a space $X$ is called semi-proclopen if it is both a semi-preopen and a semi-preclosed set.

DEFINITION 10.14. A function $f:(X, \tau) \rightarrow(Y, \sigma)$ is called perfectly contrasemi-preirresolute (written as perfectly contra-sp-irresolute) if the inverse of every semi-preopen set in $Y$ is a semi-preclopen set in $X$.

We recall the following definition.

Definition 10.15 (see [11]). A space $X$ is called semi-pre- $T_{1 / 2}$ if every gsp-closed set is semi-preclosed.

Next, we characterize the semi-pre- $T_{1 / 2}$ spaces by using the ap-sp-irresolute and ap-semi-preclosed mappings.

THEOREM 10.16. Let $(X, \tau)$ be a topological space. Then the following statements hold:

(i) $(X, \tau)$ is a semi-pre- $T_{1 / 2}$ space.

(ii) For every space $(Y, \tau)$ and every mapping $f:(X, \tau) \rightarrow(Y, \sigma), f$ is ap-sp-irresolute.

Proof. Let $F$ be a gsp-closed subset of $(X, \tau)$ and suppose that $F \subseteq f^{-1}(U)$ where $U \in \operatorname{SPO}(Y)$. Since $X$ is semi-pre- $T_{1 / 2}$ space, $F$ is a semi-preclosed, $F=\left(F_{S}\right)^{*}$. Therefore, $\left(F_{S}\right)^{*} \subseteq f^{-1}(U)$. This shows that $f$ is ap-sp-irresolute.

THEOREM 10.17. Let $(Y, \sigma)$ be a topological space. Then the following statements hold:

(i) $(Y, \sigma)$ is a semi-pre- $T_{1 / 2}$ space.

(ii) For every space $(X, \tau)$ and every mapping $f:(X, \tau) \rightarrow(Y, \sigma), f$ is apsemi-preclosed.

PROoF. The proof is similar to Theorem 10.16.

\section{REFERENCES}

[1] M. E. Abd El-Monsef, S. N. El-Deeb, and R. A. Mahmoud, $\beta$-open sets and $\beta$-continuous mapping, Bull. Fac. Sci. Assiut Univ. A 12 (1983), no. 1, 77-90.

[2] A. A. Allam, A. M. Zahran, and I. A. Hasanein, On almost continuous, $S$-continuous and set connected mappings, Indian J. Pure Appl. Math. 18 (1987), no. 11, 991-996. 
[3] D. Andrijević, Semi-preopen sets, Mat. Vesnik 38 (1986), no. 1, 24-32.

[4] P. Bhattacharyya and B. K. Lahiri, Semi-generalized closed sets in topology, Indian J. Math. 29 (1987), no. 3, 375-382.

[5] N. Biswas, On some mappings in topological spaces, Bull. Calcutta Math. Soc. 61 (1969), 127-135.

[6] _ On characterizations of semi-continuous functions, Atti Accad. Naz. Lincei Rend. Cl. Sci. Fis. Mat. Natur. (8) 48 (1970), 399-402.

[7] M. Caldas Cueva, Weak and strong forms of irresolute maps, Int. J. Math. Math. Sci. 23 (2000), no. 4, 253-259.

[8] F. Cammaroto and T. Noiri, Almost irresolute functions, Indian J. Pure Appl. Math. 20 (1989), no. 5, 472-482.

[9] S. G. Crossley and S. K. Hildebrand, Semi-closure, Texas J. Sci. 22 (1970), no. 2-3, 99-112.

[10] Semi-topological properties, Fund. Math. 74 (1972), no. 3, 233-254.

[11] J. Dontchev, On generalizing semi-preopen sets, Mem. Fac. Sci. Kôchi Univ. Ser. A Math. 16 (1995), 35-48.

[12] N. El-Deeb, I. A. Hasanein, A. S. Mashhour, and T. Noiri, On p-regular spaces, Bull. Math. Soc. Sci. Math. R. S. Roumanie (N.S.) 27(75) (1983), no. 4, 311-315.

[13] H. Kawashima, On the topological product of minimal Hausdorff spaces, TRU Math. 1 (1965), 62-64.

[14] N. Levine, Semi-open sets and semi-continuity in topological spaces, Amer. Math. Monthly 70 (1963), 36-41.

[15] _ Generalized closed sets in topology, Rend. Circ. Mat. Palermo (2) 19 (1970), 89-96.

[16] S. N. Maheshwari and S. S. Thakur, On $\alpha$-irresolute mappings, Tamkang J. Math. 11 (1980), no. 2, 209-214.

[17] A. S. Mashhour, M. E. Abd El-Monsef, and S. N. El-Deeb, On pre-continuous and weak pre-continuous mappings, Proc. Math. Phys. Soc. Egypt (1982), no. 53, 47-53.

[18] A. S. Mashhour, I. A. Hasanein, and S. N. El-Deeb, $\alpha$-continuous and $\alpha$-open mappings, Acta Math. Hungar. 41 (1983), no. 3-4, 213-218.

[19] G. B. Navalagi, Semi-preneighbourhoods and generalized semi-preregular closed sets in topological spaces, Topology Atlas Preprint \# 455.

[20] O. Njàstad, On some classes of nearly open sets, Pacific J. Math. 15 (1965), 961-970.

[21] T. Noiri, A generalization of closed mappings, Atti Accad. Naz. Lincei Rend. Cl. Sci. Fis. Mat. Natur. (8) 54 (1973), 412-415.

[22] J. H. Park and Y. B. Park, On sp-regular spaces, J. Indian Acad. Math. 17 (1995), no. 2, 212-218.

[23] I. L. Reilly and M. K. Vamanamurthy, On $\alpha$-continuity in topological spaces, Acta Math. Hungar. 45 (1985), no. 1-2, 27-32.

[24] M. K. Singal and A. R. Singal, Almost-continuous mappings, Yokohama Math. J. 16 (1968), 63-73.

[25] D. Sivaraj, Semi-homeomorphisms, Acta Math. Hungar. 48 (1986), no. 1-2, 139-145.

G. B. Navalagi: Department of Mathematics, Kle Society's, G. H. College, Haveri581110, KARNATAKA, INDIA

E-mail address: gnava1agi@ahotmai 1.com 


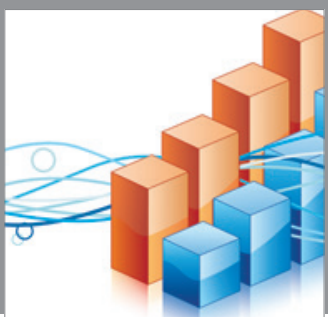

Advances in

Operations Research

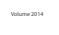

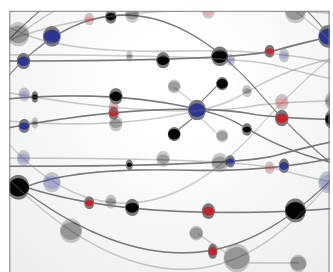

\section{The Scientific} World Journal
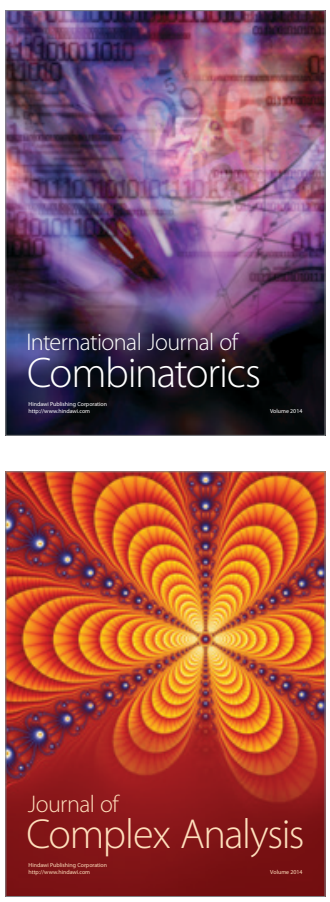

International Journal of

Mathematics and

Mathematical

Sciences
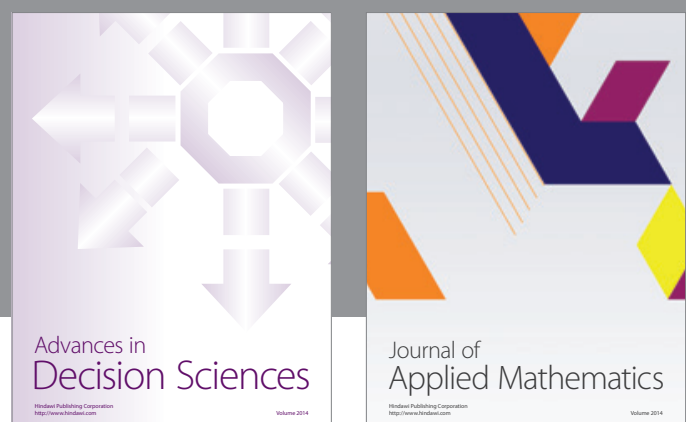

Journal of

Applied Mathematics
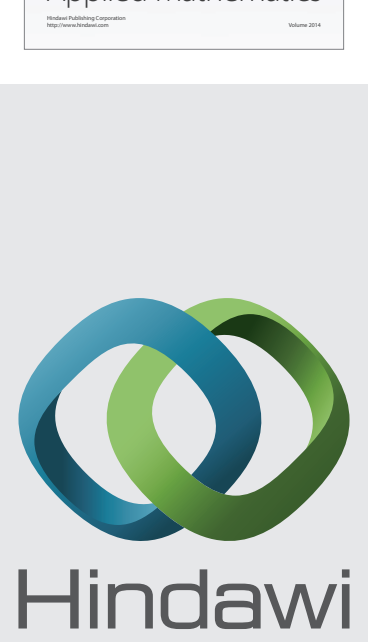

Submit your manuscripts at http://www.hindawi.com
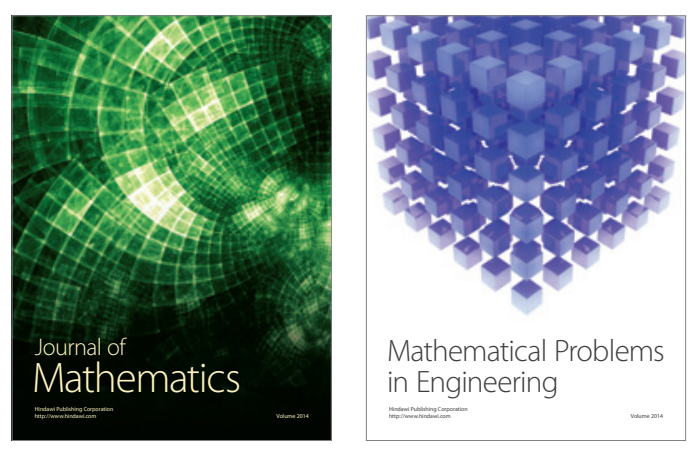

Mathematical Problems in Engineering
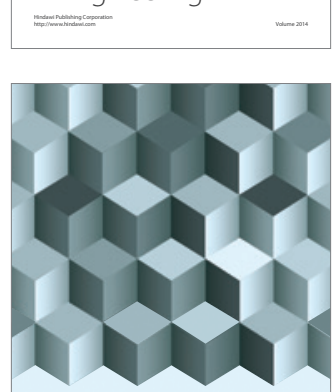

Journal of

Function Spaces
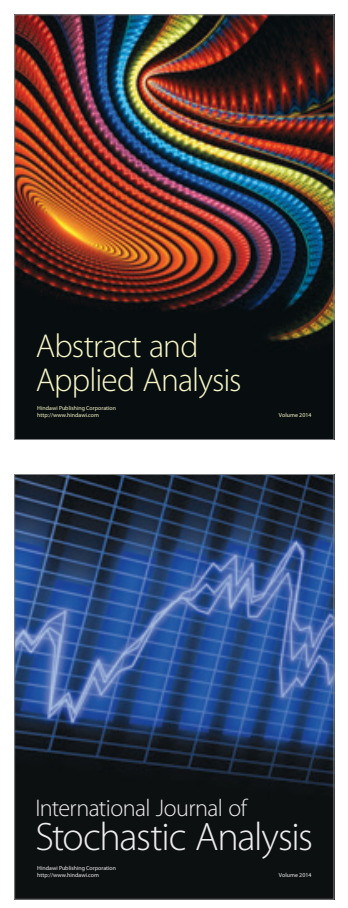

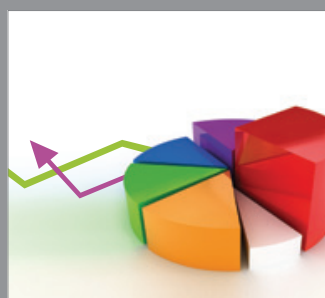

ournal of

Probability and Statistics

Promensencen
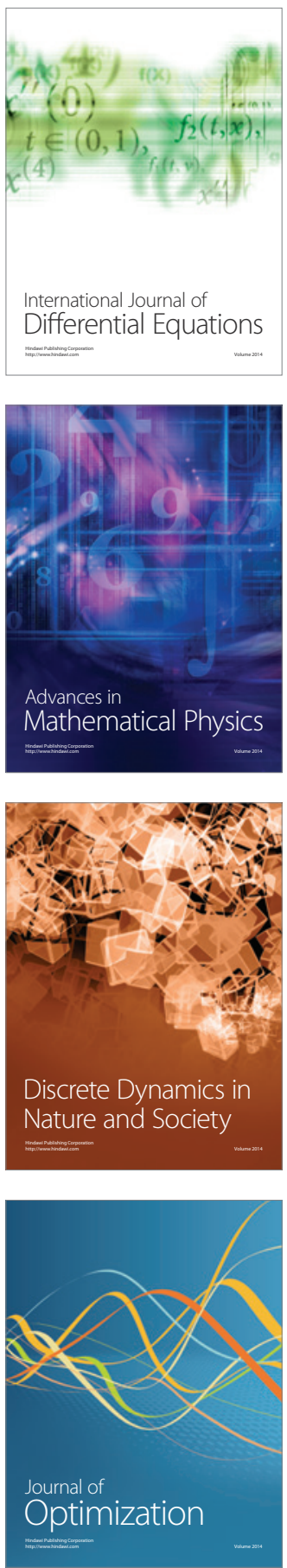